\title{
Cavity-Enhanced Raman Scattering for In Situ Alignment and Characterization of Solid-State Microcavities
}

\author{
Daniel Riedelø, ${ }^{*}$ Sigurd Flågan $\odot$, Patrick Maletinsky, and Richard J. Warburton \\ Department of Physics, University of Basel, Klingelbergstrasse 82, Basel CH-4056, Switzerland
}

(Received 26 September 2019; revised manuscript received 11 November 2019; published 22 January 2020)

\begin{abstract}
We report cavity-enhanced Raman scattering from a single-crystal diamond membrane embedded in a highly miniaturized fully tunable Fabry-Perot cavity. The Raman intensity is enhanced 58.8-fold compared to the corresponding confocal measurement. The strong signal amplification results from the Purcell effect. We show that the cavity-enhanced Raman scattering can be harnessed as a narrow-band high-intensity internal light source. The Raman process can be triggered in a simple way by using an optical excitation frequency outside the cavity stop band and is independent of the lateral positioning of the cavity mode with respect to the diamond membrane. The strong Raman signal emerging from the cavity output facilitates in situ mode matching of the cavity mode to single-mode collection optics; it also represents a simple way of measuring the dispersion and spatial intensity profile of the cavity modes. The optimization of the cavity performance via the strong Raman process is extremely helpful in achieving efficient cavity outcoupling of the relatively weak emission of single color centers such as nitrogen-vacancy centers in diamond or rare-earth ions in crystalline hosts with a low emitter density.
\end{abstract}

DOI: 10.1103/PhysRevApplied.13.014036

\section{INTRODUCTION}

The development of a quantum internet crucially relies on the scalable long-distance interconnection of quantum nodes [1]. These nodes need to combine a robust storage of quantum states and high-fidelity processing of quantum information with an efficient interface to photons mediating the network links via entanglement swapping [2]. In order to achieve high entanglement rates, these photons need to exhibit transform-limited spectral line widths, a high degree of single-photon purity, and a large creation probability per laser excitation pulse. The nitrogenvacancy $(\mathrm{N}-V)$ center in diamond constitutes a promising candidate for the stationary qubit due to its highly coherent optically addressable electron spin along with coupling to multiqubit nuclear spins in the immediate environment [3]. In seminal proof-of-principle experiments, long-distance entanglement [4] and on-demand entanglement delivery [5] between spatially separated $\mathrm{N}-V$ centers have been demonstrated. However, the entanglement rates are limited

\footnotetext{
*daniel.riedel@caltech.edu;

Current address: Institute for Quantum Information and Matter, California Institute of Technology, Pasadena, California 91125, USA.

Published by the American Physical Society under the terms of the Creative Commons Attribution 4.0 International license. Further distribution of this work must maintain attribution to the author(s) and the published article's title, journal citation, and DOI.
}

to tens of hertz due to the small fraction (approximately $3 \%$ ) of coherent photons emitted into the zero-phonon line (ZPL) [6]. A promising strategy to overcome this limitation is to enhance the ZPL photon flux of $\mathrm{N}-V$ centers via coupling to a resonant microcavity [6-9].

In recent years, tunable Fabry-Perot microcavities have been widely used to enhance the photon emission rate of various single emitters [6,10-18]. The tunability of their resonance frequency in combination with a precise lateral positioning capability allows the emitter-cavity coupling to be maximized in situ. A further advantage of this system is that micrometer-scale single-crystalline host materials can be integrated into the cavity while maintaining a high quality-factor-to-mode-volume ratio $(Q / V)$ $[6,19]$. For emitters that are highly sensitive to fluctuations of the charge environment, increasing the dimensions of a defect-free crystalline environment is clearly beneficial [20]. An example of such an emitter is the $\mathrm{N}-V$ center in diamond. $\mathrm{N}-V$ centers coupled to monolithic nanophotonic structures suffer from spectral fluctuations [8]; the optical performance is better in a Fabry-Perot microcavity [6].

To maximize their performance, tunable Fabry-Perot microcavities require precise in situ mode matching of the cavity mode to external fields. This is, in principle, simple for a well-constructed fiber mirror for which a concave mirror is fabricated at the exact center of an optical fiber [21,22]. However, this approach works well only when the mode-field diameter of the cavity is matched to that of the optical fiber. Furthermore, the mode-matching 
efficiency is inevitably limited by the different wave-front curvatures of the cavity mode and the fiber. Both mismatches are exacerbated for small cavity-mode volumes that require small mirror radii and mode-field diameters. Instead, the "top" mirror can be fabricated into a silica substrate $[6,11,14,23,24]$ and mode matching between the cavity and a single-mode fiber can be achieved with a pair of lenses. In practice, this is a nontrivial task. Mode matching is particularly difficult if two wavelengths are involved - for instance, excitation at $532 \mathrm{~nm}$ and N- $V$ ZPL emission at $637 \mathrm{~nm}$ - on account of chromatic aberrations. In these experiments, it is also desirable to measure the dispersion of the cavity modes (the dependence of the resonance frequency on the mirror separation) and the electric field distribution of each mode. These are laborious tasks if a single emitter is used.

We propose here that Raman scattering from the solidstate host is a valuable resource in aligning and optimizing tunable Fabry-Perot microcavities and in characterizing the cavity modes. The Raman scattering is enhanced by the cavity and gives large signals, facilitating quick optimization, and subsequently a simple way to determine the mode's dispersion and lateral intensity profile. The Raman scattering depends at most weakly on the lateral position, unlike a single emitter, which benefits from cavity enhancement only once the emitter is located at the cavity antinode.

Here, we report experiments on diamond from which Raman scattering is well known and has been exploited for both quantum and photonic applications. Correlated Stokes-anti-Stokes scattering in diamond [25] has led to the development of a macroscopic phonon-based quantum memory [26-29], the remote entanglement of macroscopic diamonds [30], and the development of a Raman laser in the visible-wavelength regime [31,32]. On account of the large Raman shift of diamond $\left(1332 \mathrm{~cm}^{-1}\right.$ [33]) and the high Raman gain coefficient (approximately $75 \mathrm{GW} \mathrm{cm}^{-1}$ at $532 \mathrm{~nm}$ ) [34], Stokes scattering provides an excellent narrow-line-width high-intensity internal light source. We show that cavity-enhanced Raman scattering enables fast in situ alignment of the diamond cavity mode with respect to external optics, a fast way to determine the dispersion of the cavity modes, and single-shot imaging of the lateral profiles of the modes. Additionally, comparison of the signal with cavity enhancement to the signal without the cavity is also an indicator of the single-emitter Purcell factor [35]. These attributes, demonstrated here on diamond, should be generic to single-crystal solid-state hosts.

\section{METHODS}

We create thin diamond membranes out of high-purity single-crystal diamond (Element 6), following previously reported fabrication techniques [36,37]. Using inductively coupled plasma etching and electron-beam lithography, we fabricate square-shaped membranes with a typical thickness of $1 \mu \mathrm{m}$ and side lengths of 10-50 $\mu \mathrm{m}$ [Fig. 1(a)] $[6,38]$. The membranes are bonded to a planar $\mathrm{SiO}_{2}$ substrate coated with a highly reflective distributed Bragg reflector (DBR, 15 layers of $\mathrm{SiO}_{2} / \mathrm{Ta}_{2} \mathrm{O}_{5}$, ECI evapcoat) using a micromanipulator. The extremely smooth surfaces of the diamond membrane (surface roughness $\lesssim 0.3 \mathrm{~nm}$ ) and the DBR surface promote strong adherence due to van der Waals forces. The strong bonding is demonstrated by the possibility of bending the micromanipulator needles on attempting to displace the membrane laterally [Fig. 1(a)]. The bonded membranes contain $\mathrm{N}-V$ centers, which are introduced prior to nanofabrication by nitrogen-ion implantation and subsequent annealing [6].

As a first step, we characterize the diamond membrane with a room-temperature confocal microscope (i.e., without a cavity) using an objective of high numerical aperture $(\mathrm{NA}=0.9)$. A confocal scan under continuous-wave green excitation $(\lambda=532 \mathrm{~nm}, P=580 \mu \mathrm{W})$ exhibits

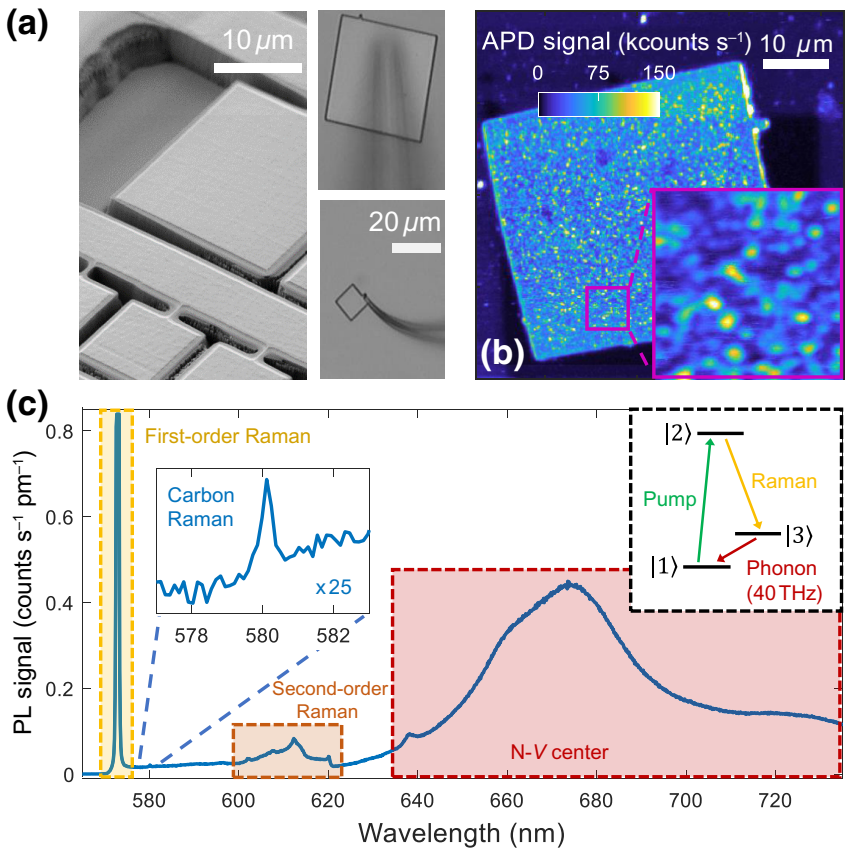

FIG. 1. (a) The fabrication and transfer of diamond membranes: left, patterning of the diamond using electron-beam lithography and inductively coupled plasma etching; right, transfer of a membrane using a micromanipulator. (b) A confocal scan of a diamond membrane under green excitation (Laser Quantum Ventus532, $\lambda=532 \mathrm{~nm}, P=580 \mu \mathrm{W}$ ) at room temperature. The emission from single N- $V$ centers can be observed. (c) The photoluminescence (PL) spectrum of a single $\mathrm{N}-V$ center at room temperature. In addition to PL, Raman features are observed: signatures of disordered carbon and first- and second-order Raman lines of the diamond lattice $(P=3.1 \mathrm{~mW}$, integration time $600 \mathrm{~s}$; for more details, see the text). The inset shows a schematic of the Stokes process, in which a pump photon is converted into a red-shifted Stokes photon and a phonon of a fixed frequency. 
well-isolated bright features, which we associate with individual N- $V$ centers [Fig. 1(b)] [39]. Figure 1(c) displays a photoluminescence (PL) spectrum for a strong excitation power $(532 \mathrm{~nm}, 3.1 \mathrm{~mW})$ and a long integration time $(600 \mathrm{~s})$, recorded at one of these bright spots. The spectrum shows a temperature-broadened N- $V$ ZPL at approximately $637 \mathrm{~nm}$ and a broad phonon side band, the spectral shape of which is slightly altered in our experiment due to the varying DBR reflectivity with wavelength and thin-film interference in the membrane. Crucially, the spectrum contains clear Raman features: the first- and second-order Stokes features at $572.67 \mathrm{~nm}$ and $600-620 \mathrm{~nm}$, respectively [33]. In addition, we find a Raman signature of carbon $s p^{2}$ bonds (Raman shift approximately $1560 \mathrm{~cm}^{-1}$ ) [40], indicating either a slight graphitic surface contamination (which could have been created during high-temperature annealing) or the presence of organic residue.

Here, we focus on the first-order Stokes scattering. This process can be modeled by a three-level atomlike system [inset Fig. 1(c)] involving a ground state $(|1\rangle)$, a virtual excited-state $(|2\rangle)$, and a metastable state $(|3\rangle)$ [31]. When the ground-state population is excited to state $|2\rangle$, it can de-excite via state $|3\rangle$ by emitting a red-shifted photon and an optical phonon of fixed energy. In our experiment, we determine a spectral shift of $\Delta E=h c \times 1335 \mathrm{~cm}^{-1}$ between the pump laser and the Stokes emission: this corresponds to the optical phonon energy in diamond.

A schematic of our tunable microcavity is shown in Fig. 2(a). A planar DBR supporting a diamond membrane (approximately $20 \times 20 \times 0.8 \mu \mathrm{m}^{3}$ ) forms a cavity with a curved DBR. We fabricate an array of atomically smooth curved microtemplates on a $\mathrm{SiO}_{2}$ chip via $\mathrm{CO}_{2}$-laser ablation, yielding small radii of curvature $[R \sim 10 \mu \mathrm{m}$, Fig. 2(b)] $[22,41]$. Subsequently, the templates are coated with a highly reflective 14-layer $\mathrm{Ta}_{2} \mathrm{O}_{5} / \mathrm{SiO}_{2}$ DBR. The spacing between the two mirrors can be adjusted by applying a voltage to the $z$ nanopositioner beneath the bottom mirror; the lateral location of the cavity's antinode can be adjusted by applying a voltage to the $x$ and $y$ nanopositioners $[6,11,14,23,24]$.

To characterize the mirrors, we measure the transmission spectrum of the planar mirror with a white-light source and quantize the data using the transmission spectrum of an uncoated quartz substrate [Fig. 2(c)]. Using a transfer-matrix calculation, we are able to reproduce the oscillations of the reflectivity over a large frequency range. A transfer-matrix-based refinement algorithm (ESSENTIAL MACLEOD) allows the reflection spectrum to be reconstructed on setting an individual-layer-thickness tolerance of $3 \%$. For our calculation, we set a stop-band center of $\lambda_{\text {center }}=625 \mathrm{~nm}$ and use $15 \lambda / 4$ layer pairs of $\mathrm{SiO}_{2}$ and $\mathrm{Ta}_{2} \mathrm{O}_{5}$ with refractive indexes of $n_{\mathrm{SiO}_{2}}=1.46$ and $n_{\mathrm{Ta}_{2} \mathrm{O}_{5}}=2.11$, respectively. For the top mirror, we obtain similar results, reproducing the transmission spectrum with $\lambda_{\text {center }}=629 \mathrm{~nm}$ and 14 layer pairs. (a)
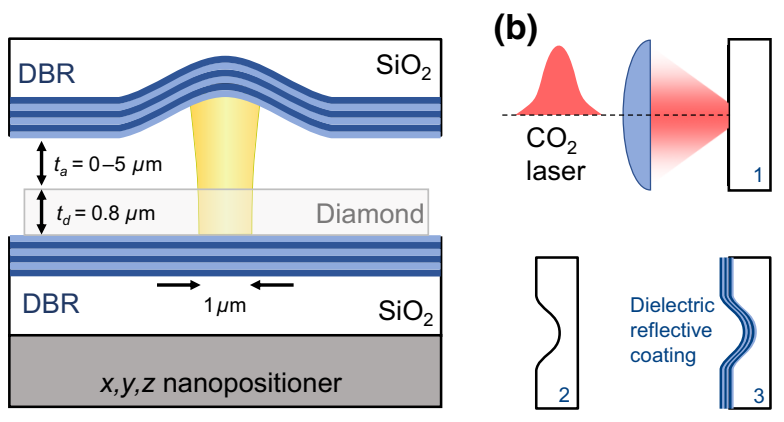

(c)

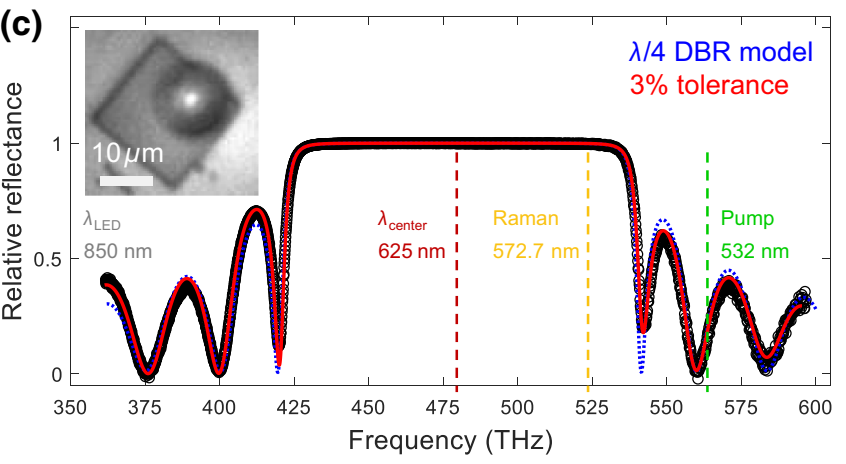

FIG. 2. (a) A schematic of a diamond membrane embedded in a tunable Fabry-Perot microcavity. Nanopositioners enable in situ control of both the resonance frequency and the antinode location of the microcavity mode. (b) The process flow of the curved microtemplate fabrication. A focused $\mathrm{CO}_{2}$ laser pulse creates a microindentation in a $\mathrm{SiO}_{2}$ substrate via ablation. Subsequently, the template is coated with a dielectric Bragg reflector (DBR). (c) The normalized white-light transmission spectrum of the DBR coating, revealing the mirror stop band. The experiment is well reproduced by a model calculation. The inset shows an image of the square-shaped membrane inside the cavity with the microindentation located on top. The bright feature in the center stems from the reflection of laser excitation at a wavelength of approximately $635 \mathrm{~nm}$.

To cavity enhance the Raman process, we pump the diamond with a green laser and tune the cavity into resonance with the Stokes line. The pump laser can be coupled into the cavity independently of the mirror separation, since its wavelength $(532 \mathrm{~nm})$ lies outside the reflection stop band of the mirror coating [Fig. 2(c)]. Conversely, due to the large Raman shift of diamond, the cavity supports a resonance with a finesse of approximately 1000 at the Stokes wavelength of approximately $573 \mathrm{~nm}$.

Some of the details of the experiment are as follows. An infrared light-emitting diode (LED, $\lambda_{\text {LED }}=850 \mathrm{~nm}$, for which the DBR is transparent) is used to locate the membrane inside the cavity [inset of Fig. 2(c)]. This visual feedback allows us to adjust the location of the diamond with respect to the top mirror. The pump laser is spectrally filtered (Semrock, LL01-532-25 and FF01-650/SP-25) and coupled into the cavity via a slightly overfilled objective of moderate numerical aperture (Microthek, $20 \times / 0.4$ ). The Stokes signal is collected via the same objective and 
coupled into a single-mode fiber (Thorlabs 630-HP); the output of this fiber is coupled into a spectrometer. A combination of a dichroic mirror (Semrock, FF560-FDi01) and long-pass and band-pass filters (Semrock, LP03-532RS-25 and FF01-572/15-25) is employed to prevent laser light entering the collection optics. This is important to avoid exciting fluorescence and Raman scattering from the detection fiber. Using a precision mechanical stage, we can move the entire microcavity with respect to the external optics, allowing the cavity output to be aligned with respect to the optical axis of the microscope.

\section{RESULTS}

Figure 3(a) shows a spectrally resolved measurement of the cavity-enhanced Stokes emission at $\lambda_{s}=572.67 \mathrm{~nm}$ pumped at $532 \mathrm{~nm}$ with $P=20 \mathrm{~mW}$. Attaining the maximal signal strength requires careful alignment. This process is massively aided by exploiting the Raman process on account of at least three factors. First, the signal is very large. When the cavity is tuned into resonance with the Stokes photons, we detect up to $2 \times 10^{6}$ photon counts $\mathrm{s}^{-1}$ on a standard silicon single-photon avalanche photodiode. Second, the signal does not depend on the $(x, y)$ alignment of the cavity: it represents a ubiquitous internal light source. Third, the Raman process couples to all the cavity modes. From the mode dispersion and the signal strengths, this allows the transverse electromagnetic (TEM) cavitymode indices $(q, n, m)$ to be determined. In particular, the $(q, 0,0)$ modes can be identified: it is these modes the output of which couples best to the single-mode fiber detection channel.

The photon flux of the cavity-enhanced Stokes scattering is strongly enhanced with respect to the Stokes signal collected from the bare diamond membrane under equivalent experimental conditions [Fig. 3(a)]. Integrating over the wavelength in both cases (with and without cavity), we find an enhancement factor of $F=58.8$. This enhancement results from the Purcell effect [42]. At a given power, the cavity increases the Stokes photon-generation rate by the Purcell factor, $F_{P}$. Additionally, the Stokes photons are emitted preferentially into the cavity mode - this enhances the detection efficiency by a factor $F_{c}$. Overall, $F=F_{P} F_{c}=58.8$. The Stokes signal increases linearly with the pump power [Fig. 3(e)]: at these pump powers, there is no superlinear dependence presaging Raman lasing.

We now exploit the Raman process as a convenient way to analyze the cavity modes. To do this, we increase gradually the cavity length, monitoring the cavity emission at the Stokes wavelength. Figure 4(a) displays the spectrally resolved measurement of the cavity emission [Fig. 3(b)] for different cavity-length detunings $\Delta L$, recorded by adjusting the width of the air gap, $t_{a}$. A series of cavity modes is observed. In this experiment, the line width
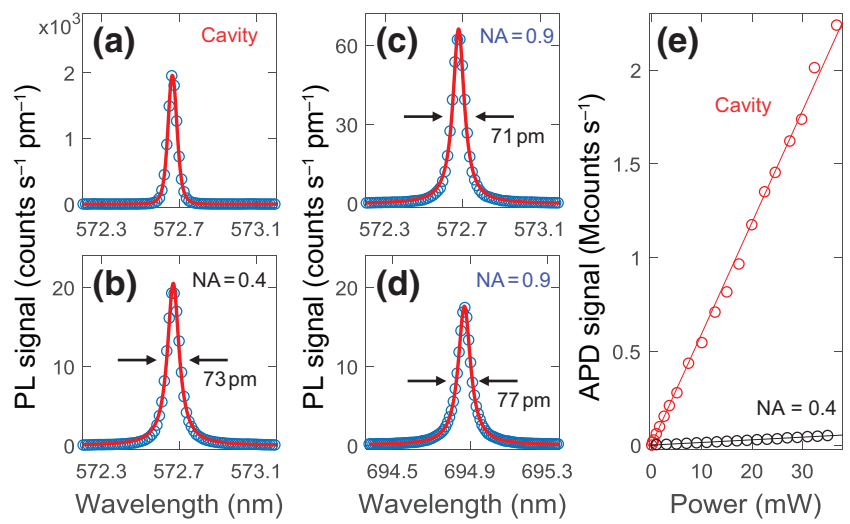

FIG. 3. (a) A typical optical spectrum of the Stokes signal in resonance with the cavity $\left(t_{\text {int }}=1 \mathrm{~s}\right)$. (b),(c) Typical optical spectra of the Stokes signal for out-of-cavity detection using objectives with (b)NA $=0.4\left(t_{\text {int }}=120 \mathrm{~s}\right)$ and (c)NA $=0.9\left(t_{\text {int }}=\right.$ $30 \mathrm{~s}$ ). (d) A typical optical spectrum of the Stokes signal pumped with a narrow-band laser at $\lambda=636 \mathrm{~nm}(P=5 \mathrm{~mW})$ without the top mirror, using an objective of NA $=0.9\left(t_{\text {int }}=180 \mathrm{~s}\right)$. (e) The power dependence of the integrated Stokes signal for outof-cavity detection with an objective of NA $=0.4$ along with the integrated cavity-enhanced Stokes signal. In (a)-(c), the pump wavelength is $\lambda=532 \mathrm{~nm}$ and the pump power $P=20 \mathrm{~mW}$; the Stokes line shape deviates slightly from a Lorentzian due to the spectral profile of the pump laser; and all data are recorded at room temperature.

of the fundamental mode $\delta t_{a}$ is a measure of the cavity finesse $\mathcal{F}=(\lambda / 2) / \delta t_{a}=350$ [Fig. 4(a), top panel] [23]. Clearly, the Raman process couples to the various higherorder Gaussian modes whenever a spectral resonance with the Stokes photons is established. The collection efficiency of the higher-order modes is much lower than that of the fundamental modes due to the signal collection through a single-mode fiber but, nevertheless, a number of higher-order modes are observed.

The exact locations of the cavity modes depend on the cavity geometry: an analysis of the spacings of the different modes allows the geometric parameters of the cavity to be determined. We extract the radius of curvature of the top mirror, $R_{\mathrm{cav}}$, from the spacings between the fundamental mode $(q, 0,0)$ and its associated higher harmonics $(q, n, m)$. Quantitatively, we make this link with a Gaussian-optics model [23]. The effective cavity length $L_{\text {eff }}, R_{\text {cav }}$, and the $(q, n, m)$ parameters are connected by

$$
L_{\mathrm{eff}}(q, n, m)=\left[q+\frac{n+m+1}{\pi} \arccos (\sqrt{g})\right] \frac{\lambda}{2},
$$

where $g$, the confocal parameter, is given by $g=1-$ $L_{\text {eff }}(q, n, m) / R_{\text {cav }} . L_{\text {eff }}$ is a measure of the separation of the two mirrors, accounting for the penetration depth into the mirrors upon reflection. From this model, we find that the modes in Fig. 4(a) are well described using $R_{\text {cav }}=$ $10 \mu \mathrm{m}$ [Fig. 4(a), lower panel]. 
(a)

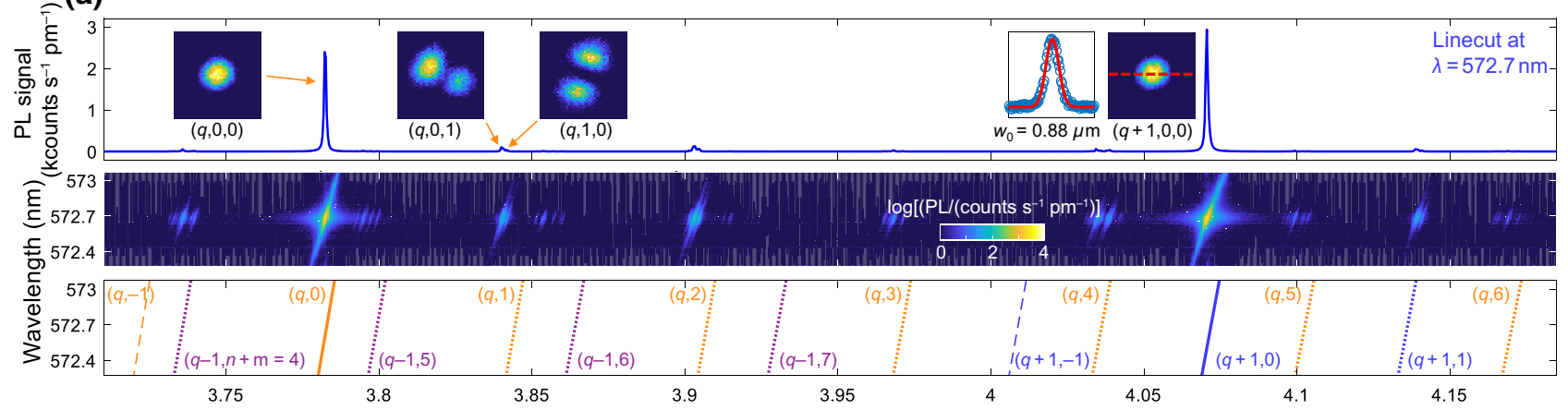

(b)
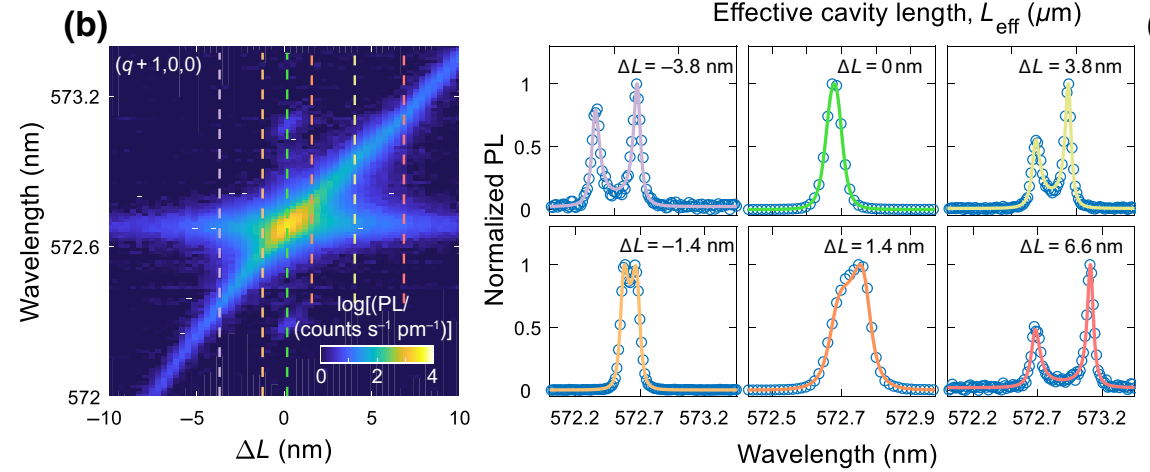

(c)

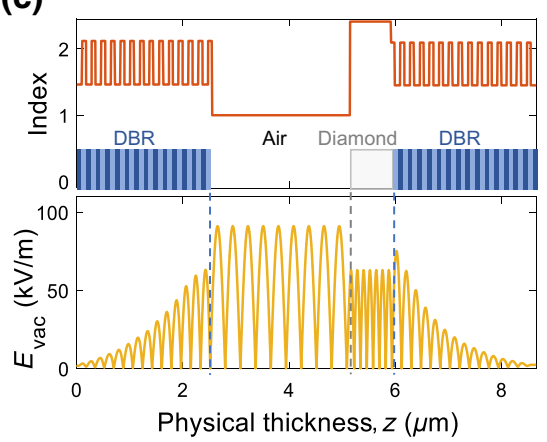

FIG. 4. (a) The center panel shows PL spectra for different relative cavity lengths $\Delta L$ about $L=4.07 \mu \mathrm{m}$ under green illumination $(\lambda=532 \mathrm{~nm}, P=20 \mu \mathrm{W})$, detected with a single-mode fiber. The top panel shows a line cut at $\lambda=572.67 \mathrm{~nm}$. The Stokes light couples to the Gaussian cavity modes $(q, n, m)$ of different mode families. Well-isolated $(q, 0,0),(q, 1,0)$, and $(q, 0,1)$ modes can be directly imaged on a CCD camera. The bottom panel shows the calculation of the mode dispersion using an analytic model based on Gaussian optics. The spacing of the different modes allows the geometric parameters of the cavity to be extracted (for details, see text). (b) An enlargement of the spectra in (a) featuring line cuts at different $\Delta L$ [for mode $(q+1,0,0)]$. The data can be fitted well using two multiplied Lorentzians (for details, see text). The data in (a) and (b) are recorded at room temperature. (c) A one-dimensional transfer-matrix simulation of the electric vacuum field for $t_{a}=2596 \mathrm{~nm}$ and $t_{d}=772 \mathrm{~nm}$.

The signals in these experiments are sufficiently large that the spatial intensity distribution of the modes can be recorded in a single-shot imaging experiment. We directly image the modal shape of the fundamental $(q, 0,0)$ and the first two higher-order modes $(q, 1,0)$ and $(q, 0,1)$ on a charge-coupled-device (CCD) camera [Fig. 4(a)]. Using the diamond membrane (edge length $20 \mu \mathrm{m}$ ) as a ruler, we can calibrate the lateral dimensions of the images. A Gaussian fit of a line cut through the fundamental mode yields a beam waist of $w_{\mathrm{I}}=0.88 \mu \mathrm{m}$ [Fig. 4(a), inset]. This value corresponds to the beam waist at the top mirror $w_{\mathrm{I}}(z=4.07 \mu \mathrm{m}, R(z)=10 \mu \mathrm{m})=0.87 \mu \mathrm{m}$, calculated from Gaussian optics.

The separation of the fundamental $(q, 0,0)$ resonances, specifically the change in resonance wavelength per change in air-gap width $m=\Delta \lambda_{c} / \Delta t_{a}$, allows the effective cavity-mode number $q$ to be inferred. For the two $(q, 0,0)$ resonances in Fig. 4(a), we measure $m_{1}=87 \mathrm{pm} \mathrm{nm}^{-1}$ and $m_{2}=83 \mathrm{pm} \mathrm{nm}^{-1}$, corresponding to $q_{1}=2 / m_{1}=23$ and $q_{2}=2 / m_{2}=24$. To interpret these numbers, the full cavity mode structure must be modeled, including the interferences induced by the diamond membrane [11]. The
Gaussian-optics model of an empty cavity [Eq. (1)] does not include these additional interferences and underestimates the actual cavity length.

Conceptually, the cavity modes can be described using a coupled-cavity approach $[6,19,43]$. In this picture, there are two cavity modes, one defined by the air gap bounded by the top DBR and the diamond-air interface; the other is defined by the diamond-air interface and the bottom DBR layer [Fig. 4(c)]. The two modes couple via the nonzero reflectivity of the diamond-air interface and hybridize. This hybridization influences the dispersion, $d \lambda_{c} / d t_{a}$, and results in deviations from the Gaussian model of an empty cavity.

We simulate the cavity modes with the aid of the same software we used to reconstruct the stop band of the DBR mirrors [Fig. 2(c)]. The exact mirror structure is included and the refractive index of diamond is assumed to be constant, $n_{d}=2.41 . m_{1}$ tells us that the lowest fundamental mode is the eighth resonance (the fifth resonance away from contact; curved mirror depth $>3 \lambda / 2)$. The gradients $m_{i}=d \lambda_{c} / d t_{a}$ of the $(q, 0,0)$ modes depend on the exact diamond thickness $t_{d}$ and the exact air-gap thickness $t_{a}$. 
By adjusting $t_{d}$ and $t_{a}$ in the simulation, we match the experimental results for $m_{1}$ and $m_{2}$ with $t_{d}=0.77 \mu \mathrm{m}$ and $t_{a}=2.60 \mu \mathrm{m}$. The diamond thickness is in agreement with the value we found in our previous $\mathrm{N}-V$ coupling experiment [6], which used the exact same membrane.

We now turn to analyzing the $(24,0,0)$ mode in more detail. This particular mode is well isolated and not perturbed by coupling to higher-order modes of other mode families [Fig. 4(b)]. Coupling between transverse modes of the cavity can be induced by a nonideal shape of the curved mirror $[44,45]$ and the fact that the diamond-air interface does not conform with the curved wave front of the Gaussian mode [19]. In particular, we focus on the spectral properties as the cavity is tuned into resonance with the Stokes process. Without the top mirror (i.e., no cavity), we find that the Stokes resonance has a Lorentzian line shape with a full width at half maximum (FWHM) of $\delta \lambda_{s, 532}=71 \mathrm{pm}$ [Fig. 3(b)]. This line width, $64.9 \mathrm{GHz}$, is determined by a convolution of the laser line width, approximately $15 \mathrm{GHz}$, with the line width of the Stokes scattering process, $\delta v_{s} \sim 50 \mathrm{GHz}$. We measure $\delta v_{s}$ independently by pumping the Stokes process with a narrow-bandwidth laser at $\lambda=636 \mathrm{~nm}$ and find $\delta \lambda_{s, 636}=$ $77 \mathrm{pm}$ corresponding to $\delta v_{s}=47.8 \mathrm{GHz}$ [Fig. 3(d)]. In the absence of inhomogeneous strain fields, the Raman line width is a measure of the phonon lifetime. The measured Raman line width corresponds well with previously reported values of 3.6-3.9 ps (40.8-44.2 GHz) [26,29], indicating low strain in our diamond membrane. Here, the main decay channel of the optical phonon involves the creation of two acoustic phonons, each with lower energy $[46,47]$. With the top mirror, we tune the cavity through the Stokes resonance, recording spectra at each detuning [Fig. 4(b)]. The experimental spectra are well fitted by the product of two Lorentzians describing the cavity and the Stokes process, $\mathcal{L}_{c}\left(\lambda_{c}, \delta \lambda_{c}\right) \mathcal{L}_{s}\left(\lambda_{s}, \delta \lambda_{s}\right)$.

During the experiment, we tune the resonance frequency of the cavity $\lambda_{c}$ while $\lambda_{s}=572.67 \mathrm{~nm}$ and $\delta \lambda_{s, 532}=71 \mathrm{pm}$ are fixed. From our fit, we extract that the line width of the cavity $\delta \lambda_{c}$ decreases from $80 \mathrm{pm}(73 \mathrm{GHz})$ to $60 \mathrm{pm}(55$ $\mathrm{GHz}$ ) on detuning from wavelength $572 \mathrm{~nm}$ to $573.4 \mathrm{~nm}$ (corresponding to $Q$ factors of 7200 and 9600, respectively) due to the change in mirror reflectivity (Fig. 2). We note that in this experiment, the bare Stokes resonance has a spectral width similar to that of the cavity resonance. On resonance, $\lambda_{c}=\lambda_{s}, \delta \lambda_{c}=70 \mathrm{pm}$, and $Q_{c \text {,res }}=8200$. The slight deviation of the Stokes line shape from a Lorentzian (see Fig. 3) results in an approximately $10 \%$ error of the extracted line width.

The spectral information leads to an interpretation of the microscopic nature of the cavity-enhanced Raman process. The spectra do not mimic the behavior of a coherent single emitter coupled to a single cavity mode. Instead, they mimic the behavior of an independent array of emitters (described by $\mathcal{L}_{s}$ ) coupled to a single cavity mode (described by $\mathcal{L}_{c}$ ). The Lorentzian cavity mode $\mathcal{L}_{c}$ acts as a spectrally selective booster for the Raman processes, which are resonant with it.

We attempt to understand the enhancement factor $F$ quantitatively [48]. The first step is to calculate the Purcell factor. One way to describe the signal enhancement promoted by the cavity is to consider an increase of the effective Stokes scattering length. The cavity finesse is a measure of the number of times a photon bounces between the mirrors and corresponds to the factor by which the scattering length is increased. This description is formally equivalent to the Purcell formula for a cavity formed by two mirrors [49]. In our experiment, however, the cavity is more complex than a generic Fabry-Perot device. We calculate the vacuum electric field distribution with the same software that we used for the previous calculations of the mirror reflectivity and the slope of the mode dispersion (ESSENTIAL MACLEOD). To that end, we calculate the electric field distribution of a one-dimensional cavity using the geometric parameters extracted from Fig. 4(a). We then quantize the field amplitude of the Gaussian cavity mode according to

$$
\begin{gathered}
\int_{\text {cav }} \epsilon_{0} \epsilon_{R}(z)\left|\vec{E}_{\mathrm{vac}}(z)\right|^{2} d z \int_{0}^{2 \pi} d \phi \int_{0}^{\infty} r e^{-r^{2} / 2 w_{\mathrm{I}}^{2}} d r \\
=2 \pi \frac{1}{4} w_{\mathrm{I}}^{2} \int_{\mathrm{cav}} \epsilon_{0} n^{2}(z)\left|\vec{E}_{\mathrm{vac}}(z)\right|^{2} d z=\hbar \omega / 2 .
\end{gathered}
$$

We take $\epsilon_{R}=n^{2}$; we assume a constant beam waist $w_{\text {I }}$ over the cavity length. Taking a representative value, $w_{0, \mathrm{I}}=$ $0.77 \mu \mathrm{m}$ (an average of the beam waist at the top mirror and the minimum beam waist), we find a maximum $\left|\vec{E}_{\mathrm{vac}}(z)\right|=54.4 \mathrm{kV} \mathrm{m}^{-1}$ inside the diamond, from which we can calculate the effective mode volume of a cubic resonator made from diamond to be $V_{\text {eff }}=84.9(\lambda / n)^{3}$. With the cavity $Q$ factor on resonance $Q_{c}\left(\lambda_{c}\right)=8200$, we calculate the Purcell factor to be $F_{P}\left(\lambda_{c}\right)=1+3 /\left(4 \pi^{2}\right) \times$ $Q_{c} / V \times(\lambda / n)^{3} \times 1 / 2=4.7$. Here, the factor of $1 / 2$ takes into account averaging of the enhancement over the field profile inside the diamond. The second step is to calculate the coupling efficiencies. Without the top mirror, we estimate the coupling efficiency $\eta_{o}$ simply as twice the solid angle defined by the objective lens: $\eta_{o} \simeq 1-$ $\sqrt{1-\left(\mathrm{NA} / n_{d}\right)^{2}}$. (The factor of 2 accounts for reflection from the bottom DBR.) With the cavity, the coupling efficiency is given by $\eta_{c}=\kappa_{t} /\left(\kappa_{t}+\kappa_{b}\right) \beta$, where $\kappa_{t}\left(\kappa_{b}\right)$ is the loss rate through the top (bottom) mirror and $\beta=$ $F_{P} /\left(F_{P}+1\right)$ is the probability of emission into the cavity mode. Assuming an identical pump rate and, likewise, identical diamond material parameters and collection optics, the ratio of the Stokes signal with cavity enhancement to the Stokes signal without the cavity (i.e., no top mirror) is $S_{c} / S_{o}=F_{P} \eta_{c} / \eta_{o}$. A final factor is that the Stokes $\left(Q_{s}\right)$ and cavity line widths $\left(Q_{c}\right)$ are similar [35]: the 


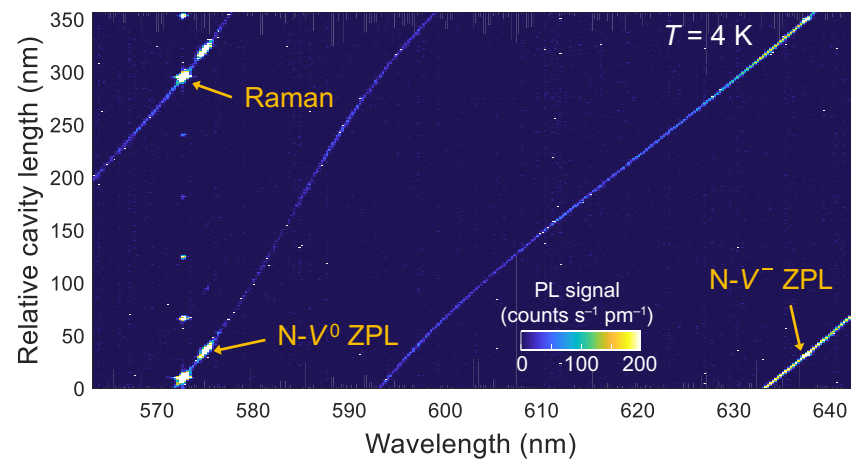

FIG. 5. The mode structure of the cavity recorded at a temperature of $4 \mathrm{~K}$, under continuous-wave excitation at $532 \mathrm{~nm}$ with a power of $30 \mathrm{~mW}$. In addition to the Raman transition, the ZPL of the $\mathrm{N}-V^{0}$ and $\mathrm{N}-V^{-}$couples to the cavity.

final result is $S_{c} / S_{o} \simeq F_{P} \times Q_{s} /\left(Q_{s}+Q_{c}\right) \times \eta_{c} / \eta_{o}$. Quantitatively, this result predicts that $S_{c} / S_{o}=56.8$, in good agreement with the experiment. (We note that the error in the experimental measurement of $S_{c} / S_{o}$ is dominated by a systematic error of $10 \%$ arising on ensuring that the optical alignment is preserved on removing the top mirror; the error in the calculated $S_{c} / S_{o}$ is dominated by the uncertainties of approximately $10 \%$ in the beam waist and the $Q$ factor of the cavity.) It is interesting to note that $S_{c} / S_{o}$ is much simpler to measure than the Purcell factor of a single emitter: a measurement of $S_{c} / S_{o}$ is an indicator of the performance of a single emitter in the same cavity structure.

The mode-structure calculation shows that the cavity is in a "diamondlike" configuration at the Stokes wavelength $\lambda_{s}$ [19]. An advantage of this configuration is that the electric vacuum field is more strongly confined in the diamond layer than in the air gap, which leads to higher coupling strengths. A further advantage is that the dispersion of the mode wavelength with the air-gap thickness is relatively small. This renders the cavity less susceptible to acoustic noise. However, a diamondlike cavity exhibits a vacuum-field antinode at the diamond-air interface, which exacerbates losses (over a configuration with a field node at the diamond-air interface) caused by scattering at this interface or absorption of the surface. In some materials - GaAs, for instance - these losses can be mitigated by passivating the surface $[24,50]$.

The results presented so far are all recorded at room temperature. For single emitters, operation at low temperature is necessary in order to eliminate phonon-related broadening of the ZPL. We therefore demonstrate that the cavity-enhanced Raman scattering also works well at cryogenic temperatures. The compact cavity design facilitates low-temperature experiments in a liquid-helium-bath cryostat $[6,14,24]$. Figure 5 shows a spectrally resolved cavity measurement where the cavity length is tuned over one free spectral range for an excitation at wavelength $532 \mathrm{~nm}$.
The weak background PL of the diamond allows the main cavity modes to be observed at all cavity lengths. In Fig. 5 , the nonlinear dependence of the cavity wavelength on the air-gap thickness is visible. The first-order Raman peak and the ZPLs of the two different charge states of the $\mathrm{N}-V$ are enhanced by the cavity and are very strong features in Fig. 5. The weak emission of the ZPLs is only detected efficiently once the external optical excitation and collection are properly aligned. The strong Raman signal is used to achieve the alignment: the advantage secured at room temperature therefore translates directly to operation at cryogenic temperatures.

\section{CONCLUSION}

We show that Raman scattering provides a valuable resource in optimizing and quantifying the performance of tunable microcavities. We apply this method specifically to the $\mathrm{N}-V$ center in diamond. More generally, the generic nature of Raman scattering renders our approach immediately applicable for improving the spin-photon interface efficiencies of a wide range of solid-state qubits. This will be particularly valuable for emitters with a weak oscillator strength: by harnessing the Stokes process as a strong narrow-band internal light source, the cavity performance can be optimized, facilitating the detection of signals from weak single emitters. Examples of qubits with long spin coherence times but small optical dipole moments include color centers in silicon carbide $[51,52]$ and rare-earth ions [53-55].

\section{ACKNOWLEDGMENTS}

We acknowledge financial support from National Centre of Competence in Research (NCCR) Quantum Science and Technology (QSIT), a competence center funded by Swiss National Science Foundation (SNF), the Swiss Nanoscience Institute (SNI), Innovative Training Network (ITN) network SpinNANO, and the European Union (EU) Quantum Flagship project ASTERIQS (Grant No. 820394).

[1] H. J. Kimble, The quantum internet, Nature 453, 1023 (2008).

[2] S. Wehner, D. Elkouss, and R. Hanson, Quantum Internet: A vision for the road ahead, Science 362, eaam9288 (2018).

[3] M. H. Abobeih, J. Cramer, M. A. Bakker, N. Kalb, M. Markham, D. J. Twitchen, and T. H. Taminiau, One-second coherence for a single electron spin coupled to a multi-qubit nuclear-spin environment, Nat. Commun. 9, 2552 (2018).

[4] B. Hensen, H. Bernien, A. E. Dréau, A. Reiserer, N. Kalb, M. S. Blok, J. Ruitenberg, R. F. L. Vermeulen, R. N. Schouten, C. Abellán, W. Amaya, V. Pruneri, M. W. Mitchell, M. Markham, D. J. Twitchen, D. Elkouss, S. Wehner, T. H. Taminiau, and R. Hanson, Experimental 
loophole-free violation of a Bell inequality using entangled electron spins separated by $1.3 \mathrm{~km}$, Nature 526, 682 (2015).

[5] P. C. Humphreys, N. Kalb, J. P. J. Morits, R. N. Schouten, R. F. L. Vermeulen, D. J. Twitchen, M. Markham, and R. Hanson, Deterministic delivery of remote entanglement on a quantum network, Nature 558, 268 (2018).

[6] D. Riedel, I. Söllner, B. J. Shields, S. Starosielec, P. Appel, E. Neu, P. Maletinsky, and R. J. Warburton, Deterministic Enhancement of Coherent Photon Generation from a Nitrogen-Vacancy Center in Ultrapure Diamond, Phys. Rev. X 7, 031040 (2017).

[7] A. Faraon, P. E. Barclay, C. Santori, K.-M. C. Fu, and R. G. Beausoleil, Resonant enhancement of the zero-phonon emission from a color center in a diamond cavity, Nat. Photonics 5, 301 (2011).

[8] A. Faraon, C. Santori, Z. Huang, V. M. Acosta, and R. G. Beausoleil, Coupling of Nitrogen-Vacancy Centers to Photonic Crystal Cavities in Monocrystalline Diamond, Phys. Rev. Lett. 109, 33604 (2012).

[9] L. Li, T. Schröder, E. H. Chen, M. Walsh, I. Bayn, J. Goldstein, O. Gaathon, M. E. Trusheim, M. Lu, J. Mower, M. Cotlet, M. L. Markham, D. J. Twitchen, and D. Englund, Coherent spin control of a nanocavity-enhanced qubit in diamond, Nat. Commun. 6, 6173 (2015).

[10] A. Muller, E. B. Flagg, M. Metcalfe, J. Lawall, and G. S. Solomon, Coupling an epitaxial quantum dot to a fiberbased external-mirror microcavity, Appl. Phys. Lett. 95, 173101 (2009).

[11] R. J. Barbour, P. A. Dalgarno, A. Curran, K. M. Nowak, H. J. Baker, D. R. Hall, N. G. Stoltz, P. M. Petroff, and R. J. Warburton, A tunable microcavity, J. Appl. Phys. 110, 053107 (2011).

[12] J. Benedikter, H. Kaupp, T. Hümmer, Y. Liang, A. Bommer, C. Becher, A. Krueger, J. M. Smith, T. W. Hänsch, and D. Hunger, Cavity-Enhanced Single-Photon Source Based on the Silicon-Vacancy Center in Diamond, Phys. Rev. Appl. 7, 024031 (2017).

[13] R. Albrecht, A. Bommer, C. Deutsch, J. Reichel, and C. Becher, Coupling of a Single Nitrogen-Vacancy Center in Diamond to a Fiber-Based Microcavity, Phys. Rev. Lett. 110, 243602 (2013).

[14] L. Greuter, S. Starosielec, A. V. Kuhlmann, and R. J. Warburton, Towards high-cooperativity strong coupling of a quantum dot in a tunable microcavity, Phys. Rev. B 92, 045302 (2015).

[15] H. Kaupp, T. Hümmer, M. Mader, B. Schlederer, J. Benedikter, P. Haeusser, H.-C. Chang, H. Fedder, T. W. Hänsch, and D. Hunger, Purcell-Enhanced SinglePhoton Emission from Nitrogen-Vacancy Centers Coupled to a Tunable Microcavity, Phys. Rev. Appl. 6, 054010 (2016).

[16] S. Johnson, P. R. Dolan, T. Grange, A. A. P. Trichet, G. Hornecker, Y. C. Chen, L. Weng, G. M. Hughes, A. A. R. Watt, A. Auffèves, and J. M. Smith, Tunable cavity coupling of the zero phonon line of a nitrogen-vacancy defect in diamond, New J. Phys. 17, 122003 (2015).

[17] D. Wang, H. Kelkar, D. Martin-Cano, T. Utikal, S. Götzinger, and V. Sandoghdar, Coherent Coupling of a Single Molecule to a Scanning Fabry-Perot Microcavity, Phys. Rev. X 7, 021014 (2017).
[18] D. Wang, H. Kelkar, D. Martin-Cano, D. Rattenbacher, A. Shkarin, T. Utikal, S. Götzinger, and V. Sandoghdar, Turning a molecule into a coherent two-level quantum system, Nat. Phys. 19, 483 (2019).

[19] E. Janitz, M. Ruf, M. Dimock, A. Bourassa, J. Sankey, and L. Childress, Fabry-Perot microcavity for diamond-based photonics, Phys. Rev. A 92, 043844 (2015).

[20] M. Ruf, M. IJspeert, S. van Dam, N. de Jong, H. van den Berg, G. Evers, and R. Hanson, Optically coherent nitrogen-vacancy centers in micrometer-thin etched diamond membranes, Nano Lett. 19, 3987 (2019).

[21] T. Steinmetz, Y. Colombe, D. Hunger, T. W. Hänsch, A. Balocchi, R. J. Warburton, and J. Reichel, Stable fiberbased Fabry-Pérot cavity, Appl. Phys. Lett. 89, 111110 (2006).

[22] D. Hunger, C. Deutsch, R. J. Barbour, R. J. Warburton, and J. Reichel, Laser micro-fabrication of concave, lowroughness features in silica, AIP Adv. 2, 012119 (2012).

[23] L. Greuter, S. Starosielec, D. Najer, A. Ludwig, L. Duempelmann, D. Rohner, and R. J. Warburton, A small mode volume tunable microcavity: Development and characterization, Appl. Phys. Lett. 105, 121105 (2014).

[24] D. Najer, I. Söllner, P. Sekatski, V. Dolique, M. C. Löbl, D. Riedel, R. Schott, S. Starosielec, S. R. Valentin, A. D. Wieck, N. Sangouard, A. Ludwig, and R. J. Warburton, A gated quantum dot far in the strong-coupling regime of cavity-QED at optical frequencies, Nature 575, 622 (2019).

[25] M. Kasperczyk, A. Jorio, E. Neu, P. Maletinsky, and L. Novotny, Stokes-anti-Stokes correlations in diamond, Opt. Lett. 40, 2393 (2015).

[26] K. C. Lee, B. J. Sussman, M. R. Sprague, P. Michelberger, K. F. Reim, J. Nunn, N. K. Langford, P. J. Bustard, D. Jaksch, and I. A. Walmsley, Macroscopic non-classical states and terahertz quantum processing in room-temperature diamond, Nat. Photonics 6, 41 (2012).

[27] D. G. England, P. J. Bustard, J. Nunn, R. Lausten, and B. J. Sussman, From Photons to Phonons and Back: A THz Optical Memory in Diamond, Phys. Rev. Lett. 111, 243601 (2013).

[28] D. G. England, K. A. G. Fisher, J.-P. W. MacLean, P. J. Bustard, R. Lausten, K. J. Resch, and B. J. Sussman, Storage and Retrieval of THz-Bandwidth Single Photons Using a Room-Temperature Diamond Quantum Memory, Phys. Rev. Lett. 114, 53602 (2015).

[29] M. D. Anderson, S. Tarrago Velez, K. Seibold, H. Flayac, V. Savona, N. Sangouard, and C. Galland, Two-Color Pump-Probe Measurement of Photonic Quantum Correlations Mediated by a Single Phonon, Phys. Rev. Lett. 120, 233601 (2018).

[30] K. C. Lee, M. R. Sprague, B. J. Sussman, J. Nunn, N. K. Langford, X. M. Jin, T. Champion, P. Michelberger, K. F. Reim, D. England, D. Jaksch, and I. A. Walmsley, Entangling macroscopic diamonds at room temperature, Science 334, 1253 (2011).

[31] A. D. Greentree and S. Prawer, A little diamond goes a long way, Nat. Photonics 4, 202 (2010).

[32] D. J. Spence, E. Granados, and R. P. Mildren, Modelocked picosecond diamond Raman laser, Opt. Lett. 35, 556 (2010). 
[33] A. M. Zaitsev, Optical Properties of Diamond (Springer, Berlin, Germany, 2010).

[34] R. P. Mildren and J. R. Rabeau, Optical Engineering of Diamond (Wiley, Weinheim, Germany, 2013).

[35] X. Checoury, Z. Han, M. El Kurdi, and P. Boucaud, Deterministic measurement of the Purcell factor in microcavities through Raman emission, Phys. Rev. A 81, 033832 (2010).

[36] P. Maletinsky, S. Hong, M. S. Grinolds, B. Hausmann, M. D. Lukin, R. L. Walsworth, M. Loncar, and A. Yacoby, A robust scanning diamond sensor for nanoscale imaging with single nitrogen-vacancy centres, Nat. Nanotechnol. 7, 320 (2012).

[37] P. Appel, E. Neu, M. Ganzhorn, A. Barfuss, M. Batzer, M. Gratz, A. Tschöpe, and P. Maletinsky, Fabrication of all diamond scanning probes for nanoscale magnetometry, Rev. Sci. Instrum. 87, 063703 (2016).

[38] D. Riedel, D. Rohner, M. Ganzhorn, T. Kaldewey, P. Appel, E. Neu, R. J. Warburton, and P. Maletinsky, Low-Loss Broadband Antenna for Efficient Photon Collection from a Coherent Spin in Diamond, Phys. Rev. Appl. 2, 064011 (2014).

[39] M. W. Doherty, N. B. Manson, P. Delaney, F. Jelezko, J. Wrachtrup, and L. C. L. Hollenberg, The nitrogen-vacancy colour centre in diamond, Phys. Rep. 528, 1 (2013).

[40] A. C. Ferrari and J. Robertson, Interpretation of Raman spectra of disordered and amorphous carbon, Phys. Rev. B 61, 14095 (2000).

[41] D. Najer, M. Renggli, D. Riedel, S. Starosielec, and R. J. Warburton, Fabrication of mirror templates in silica with micron-sized radii of curvature, Appl. Phys. Lett. 110, 11101 (2017).

[42] F. Cairo, F. De Martini, and D. Murra, QED-Vacuum Confinement of Inelastic Quantum Scattering at Optical Frequencies: A New Perspective in Raman Spectroscopy, Phys. Rev. Lett. 70, 1413 (1993).

[43] S. B. van Dam, M. Ruf, and R. Hanson, Optimal design of diamond-air microcavities for quantum networks using an analytical approach, New J. Phys. 20, 115004 (2018).

[44] J. Benedikter, T. Hümmer, M. Mader, B. Schlederer, J. Reichel, T. W. Hänsch, and D. Hunger, Transverse-mode coupling and diffraction loss in tunable Fabry-Pérot microcavities, New J. Phys. 17, 053051 (2015).
[45] D. Kleckner, W. T. M. Irvine, S. S. R. Oemrawsingh, and D. Bouwmeester, Diffraction-limited high-finesse optical cavities, Phys. Rev. A 81, 043814 (2010).

[46] P. G. Klemens, Anharmonic decay of optical phonons, Phys. Rev. 148, 845 (1966).

[47] M. S. Liu, L. A. Bursill, S. Prawer, and R. Beserman, Temperature dependence of the first-order Raman phonon line of diamond, Phys. Rev. B 61, 3391 (2000).

[48] T. Hümmer, J. Noe, M. S. Hofmann, T. W. Hänsch, A. Högele, and D. Hunger, Cavity-enhanced Raman microscopy of individual carbon nanotubes, Nat. Commun. 7, 12155 (2016).

[49] A. Reiserer and G. Rempe, Cavity-based quantum networks with single atoms and optical photons, Rev. Mod. Phys. 87, 1379 (2015).

[50] B. Guha, F. Marsault, F. Cadiz, L. Morgenroth, V. Ulin, V. Berkovitz, A. Lemaître, C. Gomez, A. Amo, S. Combrié, B. Gérard, G. Leo, and I. Favero, Surface-enhanced gallium arsenide photonic resonator with quality factor of $6 \times 10^{6}$, Optica 4, 218 (2017).

[51] D. Riedel, F. Fuchs, H. Kraus, S. Väth, A. Sperlich, V. Dyakonov, A. A. Soltamova, P. G. Baranov, V. A. Ilyin, and G. V. Astakhov, Resonant Addressing and Manipulation of Silicon Vacancy Qubits in Silicon Carbide, Phys. Rev. Lett. 109, 226402 (2012).

[52] D. J. Christle, P. V. Klimov, C. F. de las Casas, K. Szász, V. Ivády, V. Jokubavicius, J. Ul Hassan, M. Syväjärvi, W. F. Koehl, T. Ohshima, N. T. Son, E. Janzén, Á. Gali, and D. D. Awschalom, Isolated Spin Qubits in SiC with a HighFidelity Infrared Spin-to-Photon Interface, Phys. Rev. X 7, 021046 (2017).

[53] A. M. Dibos, M. Raha, C. M. Phenicie, and J. D. Thompson, Atomic Source of Single Photons in the Telecom Band, Phys. Rev. Lett. 120, 243601 (2018).

[54] T. Zhong, J. M. Kindem, J. G. Bartholomew, J. Rochman, I. Craiciu, V. Verma, S. W. Nam, F. Marsili, M. D. Shaw, A. D. Beyer, and A. Faraon, Optically Addressing Single Rare-Earth Ions in a Nanophotonic Cavity, Phys. Rev. Lett. 121, 183603 (2018).

[55] B. Casabone, J. Benedikter, T. Hümmer, F. Oehl, K. d. O. Lima, T. W. Hänsch, A. Ferrier, P. Goldner, H. de Riedmatten, and D. Hunger, Cavity-enhanced spectroscopy of a few-ion ensemble in $\mathrm{Eu}^{3}+: \mathrm{Y}_{2} \mathrm{O}_{3}$, New J. Phys. 20, 095006 (2018). 